\title{
Sub-arcsecond imaging and spectroscopic observations of the Red Rectangle in the $\mathbf{N}$-band
}

\author{
T. Miyata ${ }^{1}$, H. Kataza ${ }^{2}$, Y. K. Okamoto ${ }^{3}$, T. Onaka ${ }^{4}$, S. Sako $^{4,5}$, \\ M. Honda ${ }^{4,5}$, T. Yamashita ${ }^{5,4}$, and K. Murakawa ${ }^{5}$ \\ 1 Kiso Observatory, Institute of Astronomy, School of Science, University of Tokyo, Kiso, Nagano, Japan \\ e-mail:miyata@kiso.ioa.s.u-tokyo.ac.jp \\ 2 Institute of Space and Astronautical Science, Japan Aerospace Exploration Agency, Sagamihara, Kanagawa, Japan \\ 3 Institute of Physics, Center for Natural Science, Kitasato University, Sagamihara, Kanagawa, Japan \\ ${ }^{4}$ Department of Astronomy, School of Science, University of Tokyo, Hongo, Tokyo, Japan \\ 5 Subaru Telescope, National Astronomical Observatory of Japan, Hilo, USA
}

Received 9 July 2003 / Accepted 13 October 2003

\begin{abstract}
We carried out imaging and long-slit spectroscopic observations of the Red Rectangle in the $N$-band using the 8.2-m SUBARU telescope. The resulting images reveal a central bright core and an extended nebula. The central core is slightly extended in the $N$-band. The core radius is estimated to be $\sim 0.09^{\prime \prime}$, which corresponds to $60 \mathrm{AU}$. The core spectrum is well modeled by blackbody emission with a temperature of $358 \mathrm{~K}$. The estimated size and temperature are in good agreement with the predictions of recent dusty torus models. In the north/south nebula, unidentified infrared (UIR) emission features at $8.6 \mu \mathrm{m}$ and $11.2 \mu \mathrm{m}$ are prominently observed. At radii $>1^{\prime \prime}$ they completely dominate the $N$-band spectra, suggesting that the nebula seen at $N$-band wavelengths is primarily brightened by the UIR-band emission, not by scattered light. We also detected shifts in the UIR-feature peaks with increasing distance from the center. These may be attributed to isotopic shifts due to the presence of ${ }^{13} \mathrm{C}$ in polycyclic aromatic hydrocarbons (PAH) like particles. In addition to the UIR emission, a broad feature at $11.27 \mu \mathrm{m}$ is seen at a distance of $0.3^{\prime \prime}$ on all sides of the nebula. We attribute this to crystalline silicate olivine grains around the central torus.
\end{abstract}

Key words. circumstellar matter - stars: individual: Red Rectangle - stars: AGB and post-AGB - infrared: stars

\section{Introduction}

The Red Rectangle (AFGL915) is a well-known bipolar reflection nebula around an evolved binary system. The primary star, HD 44179, has a spectral type of A0 (Cohen et al. 1975), and its companion is considered to be a cool, luminous giant based on its spectral energy distribution (Rowan-Robinson \& Harris 1983; Men'shchikov et al. 2002). A distance to the Red Rectangle of $330 \mathrm{pc}$ has been frequently cited for over two decades, but recent model calculations suggest that $710 \mathrm{pc}$ is a more reliable estimate (Men'shchikov et al. 2002).

The nebula shows a rectangular or an $\mathrm{X}$-shaped structure extending over $40^{\prime \prime}$. The X-shaped spikes can be seen over a wide range of wavelengths from optical (e.g., Cohen et al. 1975) to mid-infrared (Hora et al. 1996 and the references therein; Waters et al. 1998). Recent optical and nearinfrared imaging observations with sub-arcsecond spatial resolution have revealed that the nebula has two lobes divided by a dark lane whose thickness is $\sim 0.15^{\prime \prime}$ at the innermost region (Roddier et al. 1995; Osterbart et al. 1997; Men'shchikov et al. 1998, 2002; Mekarnia et al. 1998; Tuthill et al. 2002;

Send offprint requests to: T. Miyata,

e-mail: miyata@kiso.ioa.s.u-tokyo.ac.jp
Murakawa et al. 2003). These structures can be accounted for by the presence of an optically thick torus (disk) and reflection lobes around the binary system. The dusty torus completely obscures the central binary system so that no direct light from the stars can be seen. The presence of a gravitationally bound torus (disk) was also inferred from the extremely narrow microwave CO emission (Jura et al. 1995). Osterbart et al. (1997) compared the extinction of the north and south lobes seen in optical wavelengths and determined an upper limit of $0.7^{\prime \prime}$ for the torus radius. Recent model calculations suggest that the radius of the torus is in the range of $0.08-0.09^{\prime \prime}$ and its hydrogen density reaches $2.5 \times 10^{12} \mathrm{~cm}^{-3}$ (Men'shchikov et al. 1998, 2002).

The Red Rectangle is known to be a strong emitter of unidentified infrared (UIR) emission features (e.g., Cohen et al. 1975) that are commonly attributed to carbonaceous materials such as polycyclic aromatic hydrocarbons (PAHs). Narrow-band imaging (Bregman et al. 1993; Hora et al. 1996) and slit spectroscopic observations (Sloan et al. 1993; Kerr et al. 1999) have revealed that carbonaceous materials are widely distributed in the nebula. The presence of the UIR features strongly indicates that the nebula has a carbon-rich environment. However, oxygen-rich materials have also been detected in the nebula. UV spectroscopy by Reese et al. (1996) 
suggested the presence of $\mathrm{OH}$ molecules. Observations by the ISO Short Wavelength Spectrometer (ISO/SWS) showed features that indicate the presence of $\mathrm{CO}_{2}$ gas and crystalline silicate (Waters et al. 1998). The fact that the $\mathrm{OH}$ and $\mathrm{CO}_{2}$ gas are observed in absorption indicates that oxygen-rich materials are located in the dusty torus. Therefore it is suggested that the crystalline silicate is distributed in the torus (Waters et al. 1998); however, direct observational confirmation has not yet been obtained.

Since the Red Rectangle nebula shows a complicated spatial structure, observations with high spatial resolution are indispensable in studying the spatial distribution of dust grains. Since the size of the dusty torus and the nebula is estimated to be 100-10000 AU, large ground-based telescopes must be used to obtain spatially resolved images or spectra. Observations in the $N$-band are particularly important because of the UIR-band features and the thermal continuum from warm dust grains that are detectable in this wavelength range. We may also find evidence for crystalline silicates, because they produce strong features in the $N$-band.

In this paper we report the results of imaging and spectroscopic observations in the $N$-band $(8-13.2 \mu \mathrm{m})$ with the SUBARU telescope. In Sect. 2 we describe the observations and the data reduction procedures. In Sect. 3 we present the images obtained and the spatially resolved spectra, In Sect. 4 we discuss the structure of the central part of the nebula (core) and the spatial distribution of dust in the nebula. The results of this study are summarized in Sect. 5 .

\section{Observations and data reduction}

We observed the Red Rectangle with the COoled MidInfrared Camera and Spectrometer (COMICS) mounted at the Cassegrain focus of the 8.2-m SUBARU telescope during 2000 8-9 December. The COMICS is a ground-based instrument that is designed for observations in the mid-infrared wavelength region $(8-26 \mu \mathrm{m})$ and has imaging and long slit spectroscopic capabilities with diffraction-limited spatial resolution. It employs five $320 \times 240 \mathrm{Si}$ :As IBC detectors for spectroscopy and one for imaging. Details of the COMICS design and performance are given in Kataza et al. (2000) and Okamoto et al. (2003).

Imaging observations were carried out with three bandpass filters in the $N$-band: $8.3-9.1 \mu \mathrm{m}(8.8 \mu \mathrm{m}$ filter $), 9.4-10.3 \mu \mathrm{m}$ (9.8 $\mu \mathrm{m}$ filter), and $12.0-13.1 \mu \mathrm{m}(12.4 \mu \mathrm{m}$ filter). The pixel scale was $0.13^{\prime \prime} /$ pixel, and the field of view was $41^{\prime \prime} \times 31^{\prime \prime}$. Secondary mirror chopping with a frequency of $0.45 \mathrm{~Hz}$ was employed in order to subtract the background radiation. The chopping throw was $20^{\prime \prime}$, yielding two on-chip images in a single chopping cycle. All sky-subtracted images were adjusted for position with an accuracy of 0.1 pix (corresponding to $0.01^{\prime \prime}$ ) and co-added to produce a final image of size $\sim 10^{\prime \prime} \times 10^{\prime \prime}$. The total on-source integration times for the $8.8,9.8$, and $12.4 \mu \mathrm{m}$ filters were $0.85,0.60$, and $1.2 \mathrm{~s}$, respectively. We observed standard stars $\beta$ Gem and $\alpha$ CMa before and after observing the Red Rectangle, for point spread function (PSF) measurements and flux calibration. The absolute fluxes of the stars were adopted from Cohen et al. (1999). The full widths at half maximum ( $F W H M$ s) of the PSF were measured to be $0.24^{\prime \prime}, 0.28^{\prime \prime}$, and $0.30^{\prime \prime}$, at $8.8,9.8$, and $12.4 \mu \mathrm{m}$, respectively.

The $N$-band spectra were obtained using the long-slit lowresolution spectroscopic mode. The slit was located on the central core position along the north-south direction $(\mathrm{N}-\mathrm{S}$ position) and the east-west direction (E-W position) (see Fig. 2). The slit position was confirmed by slit-viewer images at $8.8 \mu \mathrm{m}$ taken at the same time, and the accuracy was estimated to be as high as 0.5 pixel $\left(\sim 0.07^{\prime \prime}\right)$. The pixel size was $0.165^{\prime \prime}$. The slit width was $0.33^{\prime \prime}$, close to the diffraction-limited resolution of the SUBARU telescope in the $N$-band. A spectral resolution of $\sim 250$ was achieved at $12.8 \mu \mathrm{m}$. As in the imaging observations, secondary chopping with a frequency of $0.45 \mathrm{~Hz}$ and a throw of $20^{\prime \prime}$ was used. The obtained spectral images were corrected for geometric distortion and combined to produce an image at each slit position. The combined images cover the $10^{\prime \prime}$ field and a wavelength range of 7.8-13.4 $\mu \mathrm{m}$. The total onsource integration time was $2.7 \mathrm{~s}$ for the position NS and $4.7 \mathrm{~s}$ for the position EW. $\beta$ Gem was observed just after the observations of the Red Rectangle and used for the correction of atmospheric and instrumental transmission. Finally we co-added the spectra of two continuous rows, which corresponds to a slit width of $0.3^{\prime \prime}$, from the calibrated images, to obtain the final spectra.

\section{Results}

\subsection{Imaging}

The $N$-band images of the Red Rectangle are shown in Fig. 1. A bright core and an extended nebula are visible in all the images. Their appearance is in good agreement with the results of previous observations (Hora et al. 1996; Waters et al. 1998). The core seems to be compact, but not a point source. It is slightly extended $\left(F W H M \sim 0.4^{\prime \prime}\right)$ in all the images. Neither the elongation nor the multiplicity of the core emerges in optical and near infrared images. The detailed structure of the core will be discussed in Sect. 4.1.

The nebula shows a rectangular or an X-like shape with NE-SW and NW-SE spikes. The contrast between the nebula and the core varies slightly with wavelength: the nebula is quite striking in the $8.8 \mu \mathrm{m}$ image, whereas it is relatively faint at $9.8 \mu \mathrm{m}$.

\subsection{Spectroscopy}

Figure 3 displays the obtained $N$-band spectra. Each panel shows six spectra at positions from the core to the outer edge in each direction, and each spectrum covers a field of $0.33^{\prime \prime} \times$ $0.33^{\prime \prime}$. The spectral range where telluric ozone absorptions are strong $(9.3-10.1 \mu \mathrm{m})$ is considered unreliable, since the airmass for the Red Rectangle $(\sim 1.7)$ was much larger than for the standard $\beta$ Gem $(\sim 1.2)$. The data in this spectral region are plotted as thin dots in Fig. 3. Since they show profiles similar to the telluric ozone features, we regard them as spurious 


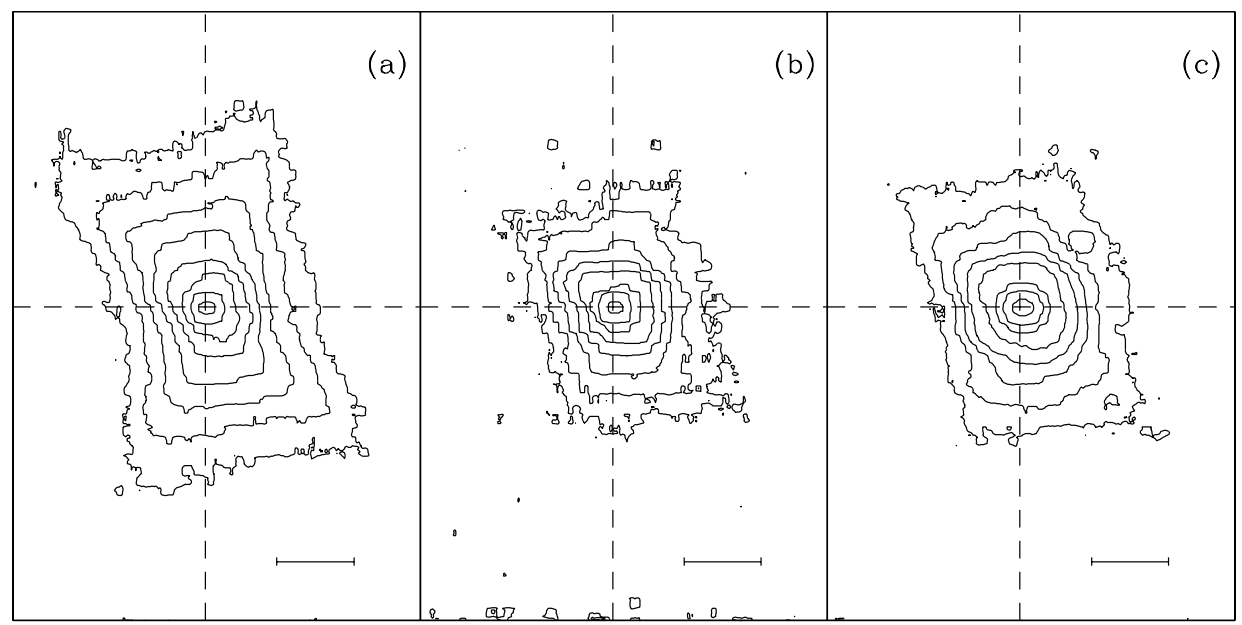

Fig. 1. Contour plots of the a) $8.8 \mu \mathrm{m}$, b) $9.8 \mu \mathrm{m}$, and c) $12.4 \mu \mathrm{m}$ images of the Red Rectangle. North is top and east is left. Contour levels are $50,25,12.5,7.25,3.13,1.56,0.78 \%$ of the peak surface brightness. Scale bars in the panels correspond to 1 arcsec.

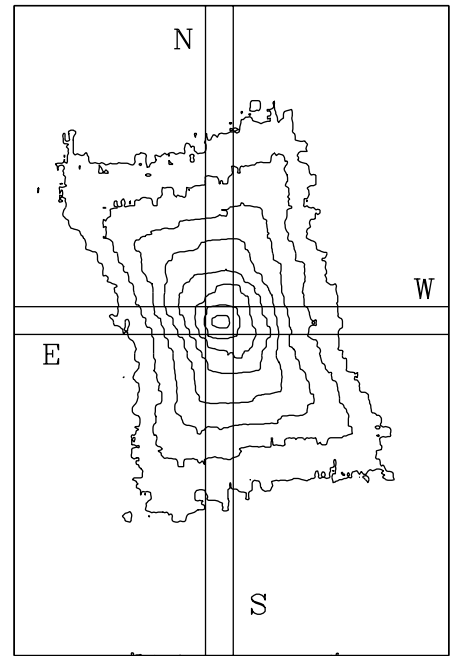

Fig. 2. Slit positions of $\mathrm{N}-\mathrm{S}$ and $\mathrm{E}-\mathrm{W}$ superimposed on the $8.8 \mu \mathrm{m}$ image.

features and will not use the spectra in this region in the following discussion.

The characteristics of the spectra vary appreciably with position. The spectra are dominated by emission features throughout the nebula, whereas continuum emission is always relatively prominent at the core. Figure 5 shows the core spectra measured at both slit positions of $\mathrm{N}-\mathrm{S}$ and $\mathrm{E}-\mathrm{W}$. They are in general agreement with each other, and show a red continuum and an emission bump at $12 \mu \mathrm{m}$. The continuum is a smooth curve increasing with wavelength. The bump at $12 \mu \mathrm{m}$ is faint relative to the continuum, but we consider it real, because it is seen in the spectra independently measured at two slit positions. This bump is detected only at the core position; at the other positions the detection is negative or only marginal.

Going away from the star, the contribution from continuum emission fades away, and the emission features stand out in the nebula region. The most prominent features are located at $8.7 \mu \mathrm{m}$ and $11.2 \mu \mathrm{m}$. They appear at a distance of $0.3^{\prime \prime}$ from the center and almost dominate the spectra beyond $1^{\prime \prime}$ in the north/south nebula. The characteristics of the features vary with distance not only in their intensities but also in their spectral profiles. An upturn in the emission at the shortest wavelengths is also discernible within $1.3^{\prime \prime}$ of the north/south nebula, including at the core position. Weak features at $12.0 \mu \mathrm{m}$ and $12.7 \mu \mathrm{m}$ are also seen beyond $0.3^{\prime \prime}$.

In the east/west nebula, on the other hand, a broad feature at $11.2 \mu \mathrm{m}$ at a distance of $\sim 0.3^{\prime \prime}$ is noticeable. The peak wavelength is about $11.3 \mu \mathrm{m}$, and the width is $0.6-0.7 \mu \mathrm{m}$. They are similar to the features seen at $0.3^{\prime \prime}$ in the north/south nebula. However, the width of the feature does not become narrower with increasing distance from the star, in contrast to the north/south nebula, and the feature almost disappears at $1.0^{\prime \prime}$. The $8.6 \mu \mathrm{m}$ feature is only marginally detected on the east/west sides. There is no evidence for the other dust features in the spectra of the east/west nebula.

There seems to be a weak feature at around $9.2 \mu \mathrm{m}$ in the spectra at $\sim 1^{\prime \prime}$ in the north and east positions. The ozone absorption between $9.3-10 \mu \mathrm{m}$ could also affect this spectral range and so we cannot confirm whether the feature is real or spurious. We will not discuss this feature in the following.

\section{Discussion}

\subsection{Core}

Figure 4 shows cuts of the core brightness in the $N$-band along the polar (a position angle of $104 \mathrm{deg}$, left panels) and the disk (14 deg, center panels) directions. The FWHMs of the core were measured to be $0.37^{\prime \prime}, 0.37^{\prime \prime}$, and $0.42^{\prime \prime}$ at $8.8 \mu \mathrm{m}$, $9.8 \mu \mathrm{m}$, and $12.4 \mu \mathrm{m}$, respectively. They are certainly larger than the PSFs (shown by crosses in Fig. 4). No clear differences can be found in the two directions, suggesting that the core has a circular structure in the $N$-band.

The measured FWHMs are affected by the PSFs of the observing system and they can be regarded as a convolution of intrinsic brightness distributions with the PSFs. We created model brightness profiles by convolving Gaussian functions with the PSFs and compared them with the observed profiles 

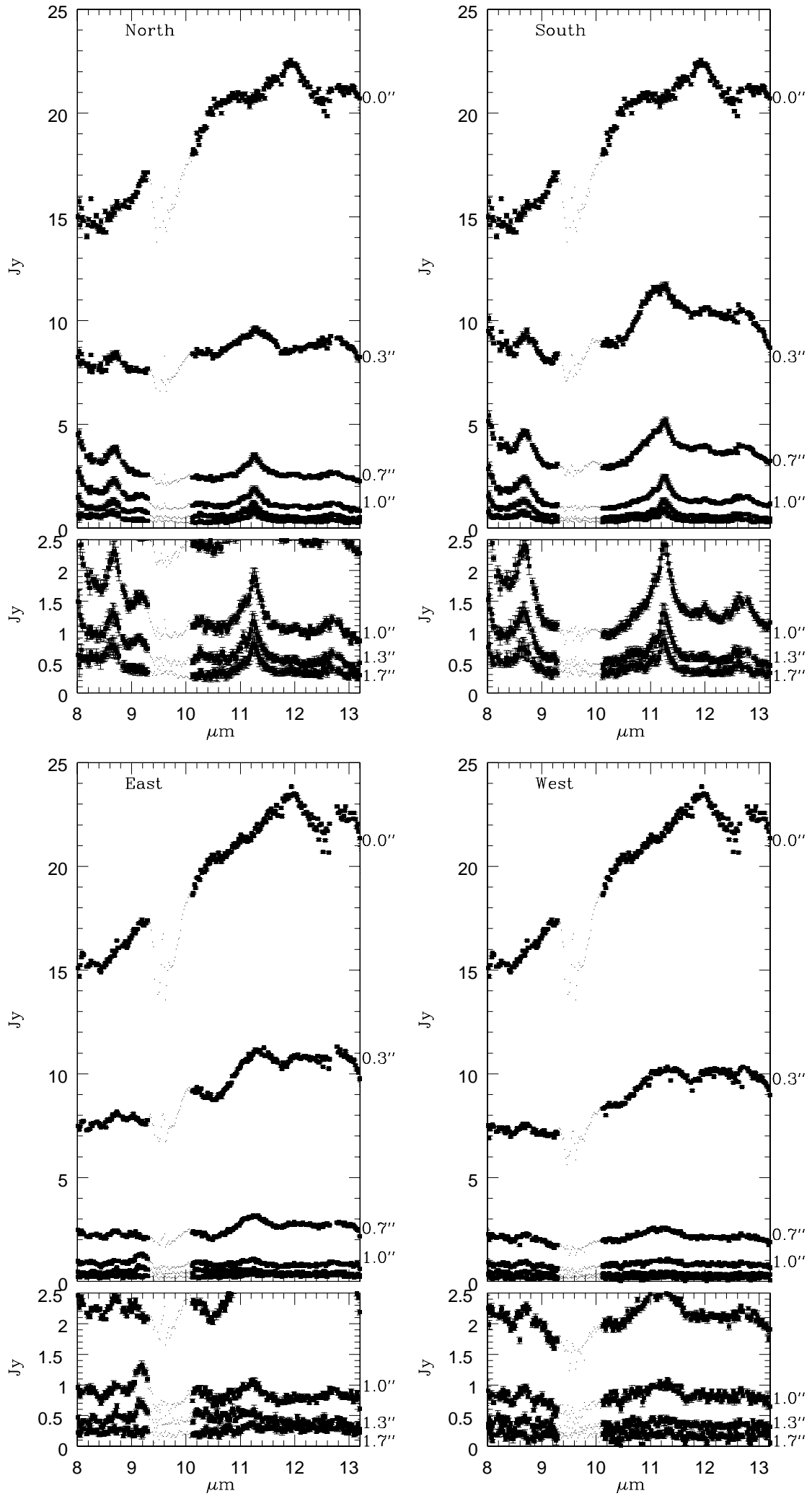

Fig. 3. Spatially resolved spectra of the Red Rectangle. Each spectrum covers a field of $0.3^{\prime \prime} \times 0.3^{\prime \prime}$. Lower small panels are enlargements of the spectra. The spectra are not offset. Upper left: spectra from center to the north. Upper right: to the south. Lower left: to the East. Upper right: to the West.

to estimate the true size of the torus. The observed profiles of the standard stars were used as the PSFs. We found that Gaussian functions with FWHM of $0.18^{\prime \prime}$ can account for the measured $F W H M$ s most reasonably at all wavelengths when convolved with the PSFs (see right panels in Fig. 4). Therefore the radius of the torus is estimated to be $\sim 0.09^{\prime \prime}$, which 


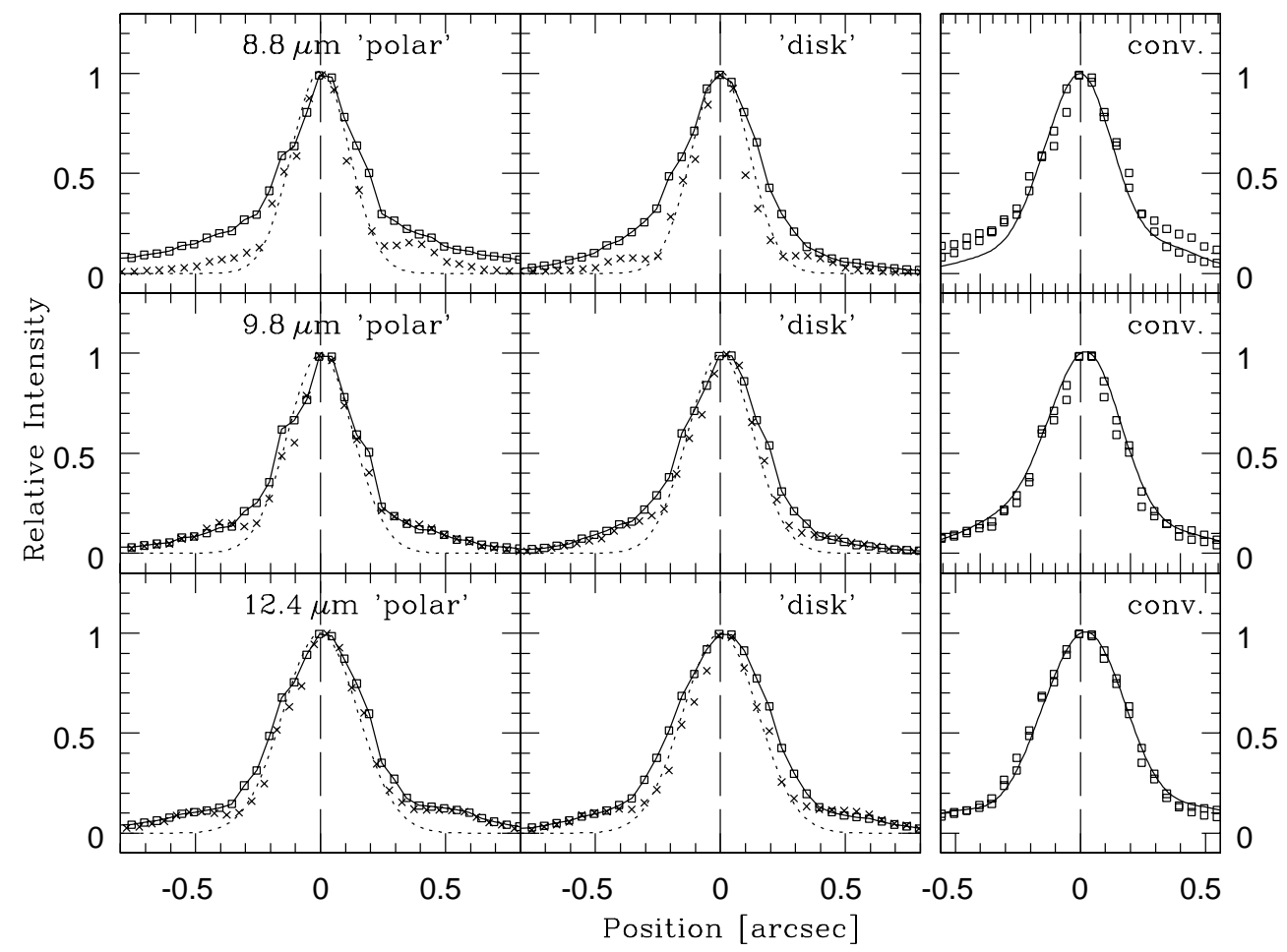

Fig. 4. Brightness distributions of the (upper to lower) $8.8 \mu \mathrm{m}, 9.8 \mu \mathrm{m}$, and $12.4 \mu \mathrm{m}$ images at the core. Left panels: the distribution along the "polar" direction (position angle $=104^{\circ}$ ). South is left and north is right. The squares connected by lines show the intensity profile of the Red Rectangle. The crosses are the PSFs of the standard stars. The dotted line shows the Gaussian function with FWHMs of $0.24^{\prime \prime}, 0.26^{\prime \prime}, 0.30^{\prime \prime}$ for $8.8 \mu \mathrm{m}, 9.8 \mu \mathrm{m}$, and $12.4 \mu \mathrm{m}$, respectively. Center panels: same as the left panels, but along the "disk" direction (the position angle $=14^{\circ}$ ). East is left and west is right. Right panels: Gaussian function with FHWM of $0.18^{\prime \prime}$ convolved with the PSF.

corresponds to $60 \mathrm{AU}$ at a distance of $710 \mathrm{pc}$. No clear excess around the wings of the PSF can be seen in the $9.8 \mu \mathrm{m}$ and $12.4 \mu \mathrm{m}$ profiles, suggesting that emission from outside the torus, such as scattered light from the nebula, does not contribute significantly in the innermost region.

To estimate the temperature of the torus, we extracted the data points in the ranges of 8.5-9.0, 10.1-11.0, and $12.4-12.6 \mu \mathrm{m}$ from the spectrum at the core, and fitted them with a single-temperature blackbody model. The best-fit temperature was $358 \mathrm{~K}$, consistent with that obtained by Sloan et al. (1993).

The estimated size and temperature of the core are in good agreement with the predictions of recent dusty torus models. Men'shchikov et al. (2002) made two-dimensional radiative transfer calculations for a compact, axisymmetric, edge-on circumbinary torus model and successfully accounted for both the high-resolution images in optical and near-infrared regions as well as the spectral energy distribution from UV to radio. Their model predicts that the torus consists primarily of millimeter sized grains with gray emissivity and their temperature goes down to $358 \mathrm{~K}$ at a distance of $\sim 0.07 "$ ( $50 \mathrm{AU})$. This is consistent with our results. The model also predicts that a large number of grains with higher temperatures exist in the innermost part of the torus; however, we find no indications of the presence of high-temperature grains in our spectrum. Since the density gradient of the torus is extremely steep within $100 \mathrm{AU}$, and the torus is not geometrically thin, grains in the inner region should be completely obscured and invisible in the $N$-band.
In the spectrum of the core we can clearly see the $12 \mu \mathrm{m}$ bump emission in addition to the thermal continuum. It is a weak feature, and the feature-to-continuum flux ratio is $\sim 0.05$ at its peak wavelength. This bump is observed only at the core position and not detected at other positions, suggesting that the material from which it originates is distributed only in the dusty torus. The nature of this carrier material remains an open question. The UIR-band family has a feature around $12.0 \mu \mathrm{m}$, which is attributed to a $\mathrm{CH}$ bending mode for duo hydrogen on the $\mathrm{C}$ skeleton. This feature was detected in the Red Rectangle by previous observations (Witteborn et al. 1989; Hony et al. 2001; van Diedenhoven et al. 2003). We do not believe, however, that this assignment is correct, because the $12.0 \mu \mathrm{m}$ feature is usually much weaker than the other features at $11.23 \mu \mathrm{m}$ and $12.7 \mu \mathrm{m}$, which are not seen in our spectra. Some post-asymptotic giant branch (AGB) stars which show the $21 \mu \mathrm{m}$ emission also show abnormal features in the $10 \mu \mathrm{m}$ region, but their profiles are too broad to explain the bump. Further observational and experimental studies are needed to identify the carrier material from which this emission originates.

\subsection{Dust in the nebula}

As mentioned in Sect. 3, the continuum emission weakens with distance from the center, and the emission features become more prominent in the north/south nebula. The most prominent features peak at $8.7 \mu \mathrm{m}$ and $11.2 \mu \mathrm{m}$. To investigate the 


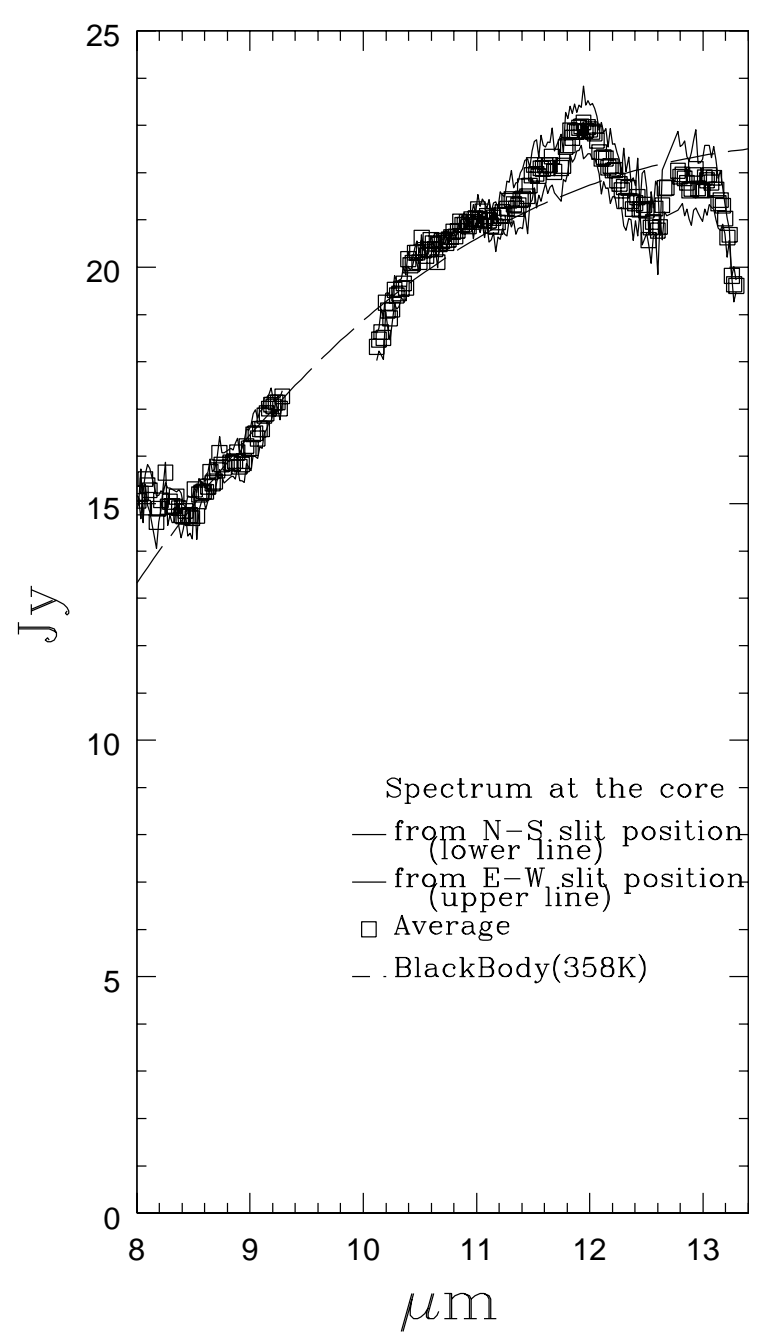

Fig. 5. Spectrum at the core. Two thin lines show independent measurements for the N-S (lower) and E-W (upper) slit positions. The averaged spectrum is plotted as open rectangles. The dashed line indicates a blackbody emission of $358 \mathrm{~K}$ (see text).

profiles of the $8.7 \mu \mathrm{m}$ and $11.2 \mu \mathrm{m}$ features in detail, we drew a straight line between the edges of the features for each band and estimated the feature profiles above the lines at each position. The straight continua are defined by the data in the ranges $10.5-10.7 \mu \mathrm{m}$ and $11.7-11.9 \mu \mathrm{m}$ for the $11.2 \mu \mathrm{m}$ feature, and $8.2-8.4 \mu \mathrm{m}$ and $8.9-9.1 \mu \mathrm{m}$ for the $8.7 \mu \mathrm{m}$ feature. Continuum-subtracted features are shown in Fig. 6.

The feature profiles vary with distance. In the outer part of the north/south nebula, the $11.2 \mu \mathrm{m}$ feature shows a sharp profile. The width of the feature is approximately $0.2 \mu \mathrm{m}$, and its peak is located between 11.23 and $11.27 \mu \mathrm{m}$. The profiles of the feature are similar to each other in the region $>0.3^{\prime \prime}$. On the other hand, towards the center, the profile of the $11.2 \mu \mathrm{m}$ feature suddenly broadens, at a distance of $0.3^{\prime \prime}$. Similar features can also be seen at the same distance in the east/west nebula. This feature clearly differs from the feature seen in the outer region. We discuss the broader feature seen at $0.3^{\prime \prime}$ and the sharp one in the outer region separately in the following.

Scarrott et al. (1992) indicated that there is sharpening of the unidentified optical-band emission features with distance over a much larger scale $\left(>5^{\prime \prime}\right)$. Since we do not see variation in the band width over this spatial scale (see next subsection), the band profile variation in the optical and infrared are likely to be of different origins.

\subsubsection{Outer region $\left(>0.3^{\prime \prime}\right)$}

In the outer region, the spectra are nearly dominated by the sharp emission peaking at 8.7 and $11.2 \mu \mathrm{m}$. Their peak wavelengths and the widths are in good agreement with UIR-band emission. Previous studies pointed out the possibility that the nebula could be illuminated by scattered light in the $N$ band on scales out to 15" (Tuthill et al. 2002; Men'shchikov et al. 2002). However, Fig. 3 clearly demonstrates that the UIR emission provides $>90 \%$ of the total flux at distances $>1$ ". We conclude that the nebula outside $1^{\prime \prime}$ is brightened mainly by the emission from the UIR bands, rather than by scattered light.

Although the UIR features seen in the outer region appear initially similar to each other, their peak wavelength moves to shorter wavelengths with increasing distance from the central object. Figure 7 shows the peak shift in the $8.6 \mu \mathrm{m}$ and $11.2 \mu \mathrm{m}$ features from a distance of $0.7^{\prime \prime}$ to $1.7^{\prime \prime}$. The peak wavelength of the $8.6 \mu \mathrm{m}$ feature moves from $8.70 \mu \mathrm{m}$ at $0.7^{\prime \prime}$ (500 AU) to $8.65 \mu \mathrm{m}$ at $1.7^{\prime \prime}$ (1200 AU). The $11.2 \mu \mathrm{m}$ feature shifts from $11.27 \mu \mathrm{m}$ to $11.23 \mu \mathrm{m}$ as well. The wavelength calibration was carried out by comparison with atmospheric lines that were observed together with the target spectra, and is considered to be accurate to $\sim 0.005 \mu \mathrm{m}$. We therefore conclude that the observed shifts are real. Shifts in the peak wavelengths of the $7.7 \mu \mathrm{m}$ and $11.2 \mu \mathrm{m}$ emissions in the nebula have also been reported by Song et al. (2003). The widths of the features are essentially unchanged with distance.

Hony et al. (2001) and van Diedenhoven et al. (2003) analyzed the UIR emission in many astronomical objects obtained by the ISO/SWS and showed that in the majority of cases the $11.2 \mu \mathrm{m}$ feature peaks at $11.23 \mu \mathrm{m}$. This is consistent with the presented spectra at $1.7^{\prime \prime}$, suggesting that the material producing the UIR emission in the outer nebula has similar characteristics to the normal band carriers in the interstellar medium (ISM). In the central region (within about $0.7^{\prime \prime}$ ), however, the UIR carriers differ from this. We note that the $11.2 \mu \mathrm{m}$ feature of the Red Rectangle measured by the ISO/SWS is slightly shifted to longer wavelengths (van Diedenhoven et al. 2002; Wada et al. 2003). A shift in the $8.6 \mu \mathrm{m}$ feature has also been reported by Peeters et al. (2002). The peak wavelengths of the $11.2 \mu \mathrm{m}$ and $8.6 \mu \mathrm{m}$ features in the SWS spectrum are $11.24 \mu \mathrm{m}$ and $8.67 \mu \mathrm{m}$, respectively. They lie at midpoints between the peak wavelengths of the features observed at $0.7^{\prime \prime}$ and $1.7^{\prime \prime}$. The ISO/SWS results can be explained by a flux-weighted mean of the shifted UIR emission in the inner region and the normal UIR emission in the outer region, since the ISO/SWS had a relatively large aperture $\left(14^{\prime \prime} \times 20^{\prime \prime}\right)$.

What causes the shifted UIR emission in the inner region? One possible explanation is an enhancement of the isotopic carbon $\left({ }^{13} \mathrm{C}\right)$ fraction in the band carriers. Recently Wada et al. (2003) made an experimental study of ${ }^{13} \mathrm{C}$ isotope 

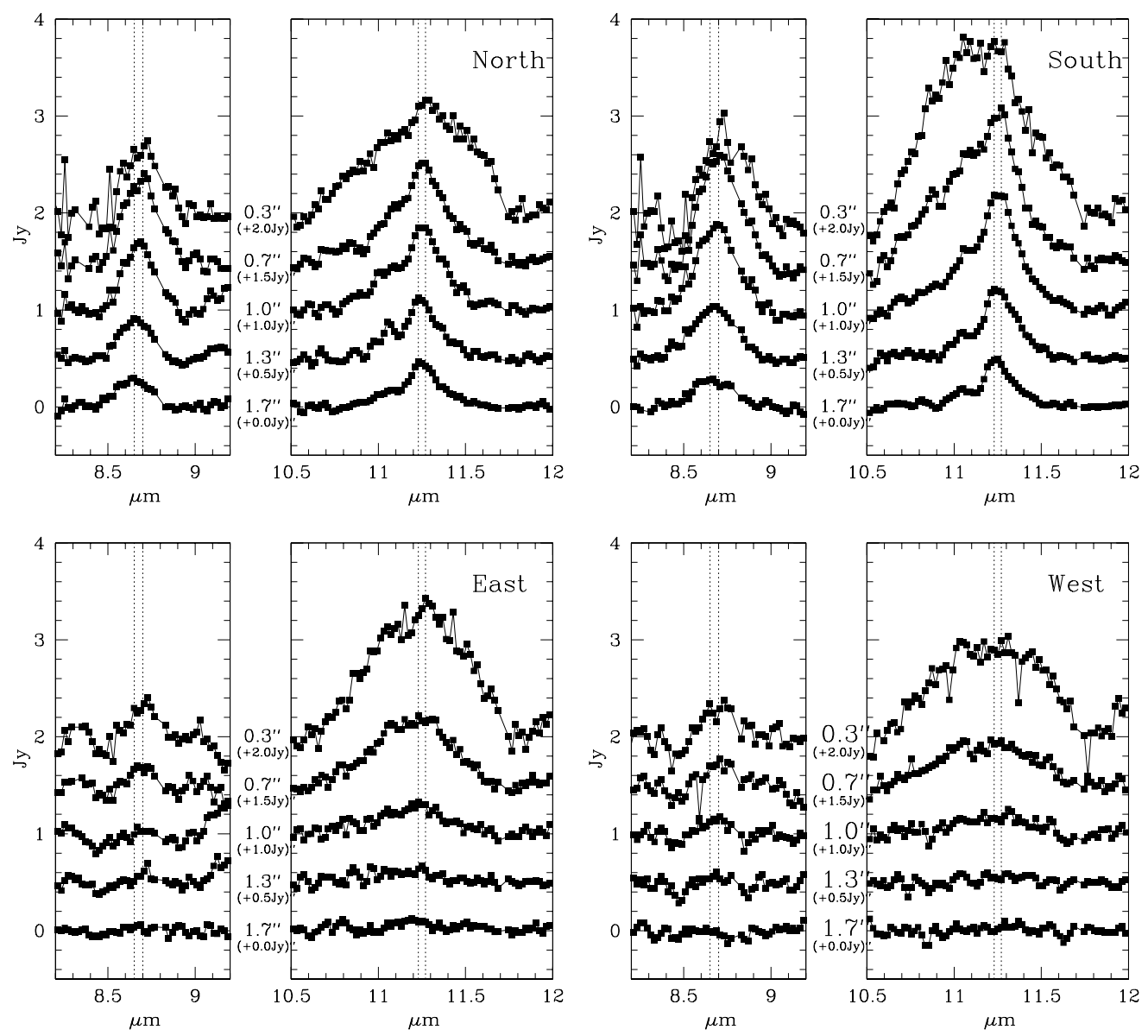

Fig. 6. Continuum subtracted spectra of the UIR emission at $0.3^{\prime \prime}, 0.7^{\prime \prime}, 1.0^{\prime \prime}, 1.3^{\prime \prime}$, and $1.7^{\prime \prime}$. Vertical dotted lines indicate the wavelengths of $8.65,8.70,11.23$, and $11.27 \mu \mathrm{m}$, respectively. The spectra are shifted to avoid overlapping.

effects on the infrared bands of a laboratory analogue of carbonaceous dust, the quenched carbonaceous composite (QCC; Sakata et al. 1984). They found that the peak wavelength of the $11 \mu \mathrm{m}$ band increases almost linearly with the ${ }^{13} \mathrm{C}$ fraction. The shift is large $\left(\Delta \lambda=0.16 \mu \mathrm{m}\right.$ per ${ }^{13} \mathrm{C}$ fraction in the total number of $\mathrm{C}$ ) enough to explain the present shift of $\Delta \lambda=0.04 \mu \mathrm{m}$, although the peak wavelength of the QCC emission $(11.4-11.6 \mu \mathrm{m})$ does not match that of the observed band. The abundance of ${ }^{13} \mathrm{C}$ relative to ${ }^{12} \mathrm{C}$ changes drastically in the final stage of stellar evolution. It increases during the first dredge-up on the red giant branch, as the convective envelope reaches regions abundant in ${ }^{13} \mathrm{C}$. In the third dredge-up during the $\mathrm{AGB}$ phase, ${ }^{13} \mathrm{C}$ is generally expected to become less abundant, but it could also increase due to cool bottom processing for low-mass stars or to hot bottom burning for more massive stars (Balser et al. 2002). Waters et al. (1998) indicated relatively strong ${ }^{13} \mathrm{CO}$ emission in the $4.6 \mu \mathrm{m}$ band for the Red Rectangle. Since the spatial distribution of dust grains records the history of the stellar evolution, spatial variation in the ${ }^{13} \mathrm{C}$ to ${ }^{12} \mathrm{C}$ ratio should occur. Assuming an expansion velocity of $10 \mathrm{~km} \mathrm{~s}^{-1}$, the angular extent of the change in the spatial distribution of the $11.2 \mu \mathrm{m}$ feature corresponds to 250-350 years; i.e., the time scale of the isotopic variation, if any, must be $\sim 300$ years.

\subsubsection{Near the torus $\left(\sim 0.3^{\prime \prime}\right)$}

The broad $11.2 \mu \mathrm{m}$ feature is seen not only in the north/south nebula but also in the east/west nebula. To examine the profile of the feature, we co-added the four spectra observed at $0.3^{\prime \prime}$ for the north, south, east, and west sides. The co-added spectrum clearly shows a peak around $11.27 \mu \mathrm{m}$ and a small bump at $12.0 \mu \mathrm{m}$ (Fig. 8). The width of the $11.27 \mu \mathrm{m}$ feature is $0.6 \mu \mathrm{m}$, which is three times wider than that of the UIR feature observed in the outer region. Therefore we conclude that the broad $11.27 \mu \mathrm{m}$ feature is different from the UIR emission. Other dust components must contribute to this feature.

The most likely candidate for the carrier material is crystalline olivine. Waters et al. (1998) found a number of emission features in the wavelength range $19-45 \mu \mathrm{m}$ and suggested that crystalline silicate grains exist in the (outer part of the) central circumbinary torus. Its temperature has been determined to be $\sim 120 \mathrm{~K}$ from the intensity ratios of the 23.75 and $33.85 \mu \mathrm{m}$ bands (Waters et al. 1998). Since crystalline silicate also has an evident feature at $11.3 \mu \mathrm{m}$ (Koike et al. 1993), it is expected that emission at $11.3 \mu \mathrm{m}$ should be seen in the $N$-band spectra (Fig. 1 of Waters et al. 1998). Based on the derived temperature and the strength in the 23.75 and $33.85 \mu \mathrm{m}$ bands, the peak flux at $11.3 \mu \mathrm{m}$ is estimated to be $6 \mathrm{Jy}$, assuming that the feature is resolved in our spectra and that the carriers are not distributed 


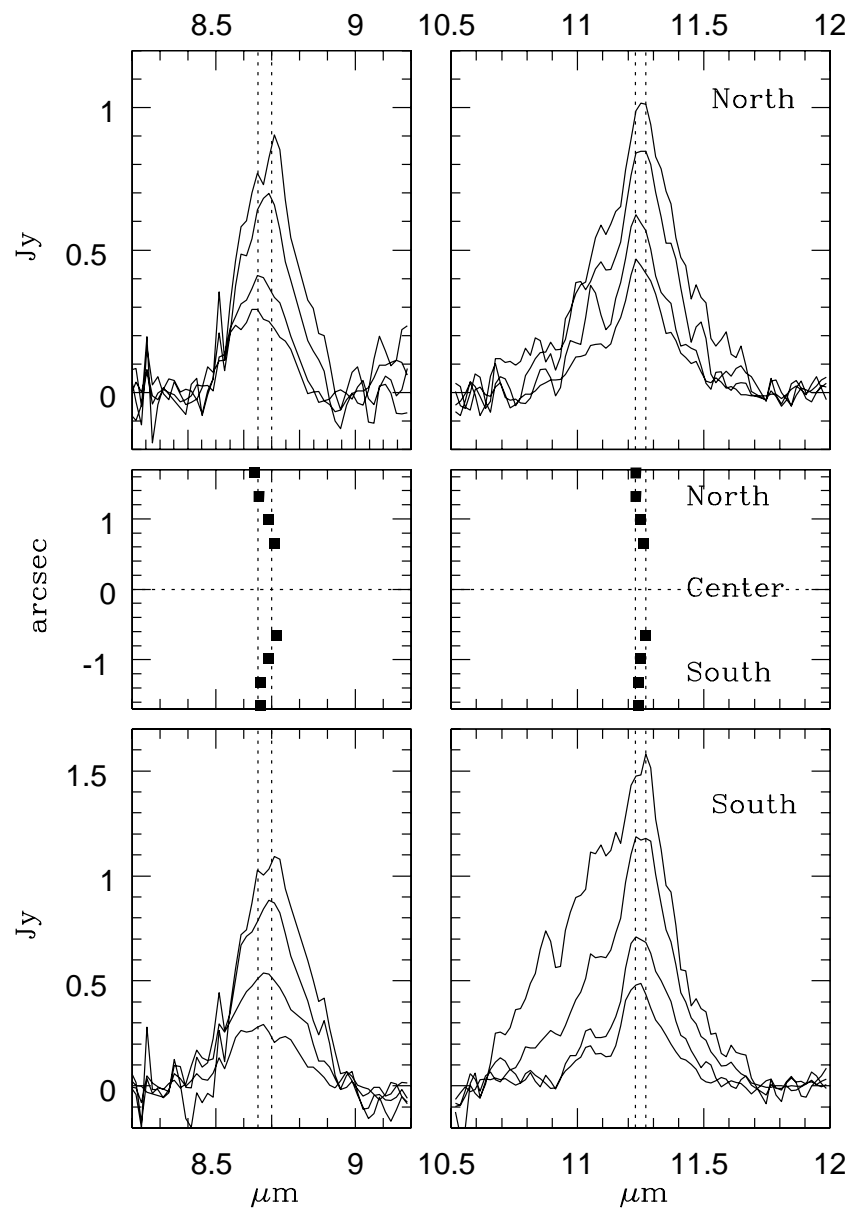

Fig. 7. Comparison of the UIR features in the outer nebula $\left(0.7^{\prime \prime}-1.7^{\prime \prime}\right)$. The spectra shown here are the same as in Fig. 6, but are not shifted. Upper panels: features in the north side of the nebula. Lower panels: same as the upper panels, but in the south side. Center panels: shift of the peaks. The filled rectangles indicate the peak positions. The peak positions clearly move to shorter wavelengths as distance increases.

widely in the nebula. The expected olivine feature is also plotted in Fig. 8. This indicates a good agreement with the co-added spectrum not only in the width and peak wavelength of the feature but also in the band flux. It suggests that most crystalline silicate grains detected by ISO are concentrated around the torus.

It is noteworthy that the feature is seen only around the core but is undetected at the core position itself (see Fig. 5). This suggests that crystalline silicate emitting the $11.2 \mu \mathrm{m}$ feature is present only in the low-density region outside the torus ( $200 \mathrm{AU})$, and does not exist in the torus. Previous studies have suggested that grains in the torus can grow to submillimeter sizes (Jura et al. 1997; Men'shchikov et al. 1998). Large grains with sizes $>10 \mu \mathrm{m}$ cannot effectively emit the features in the $N$-band. Therefore the absence of a crystalline silicate feature from the core may be explained if most crystalline silicate is locked up in large grains in the dense torus. To elucidate the distribution of the crystalline silicate clearly, spatially resolved spectroscopy in the $19-45 \mu \mathrm{m}$ wavelength range is required.

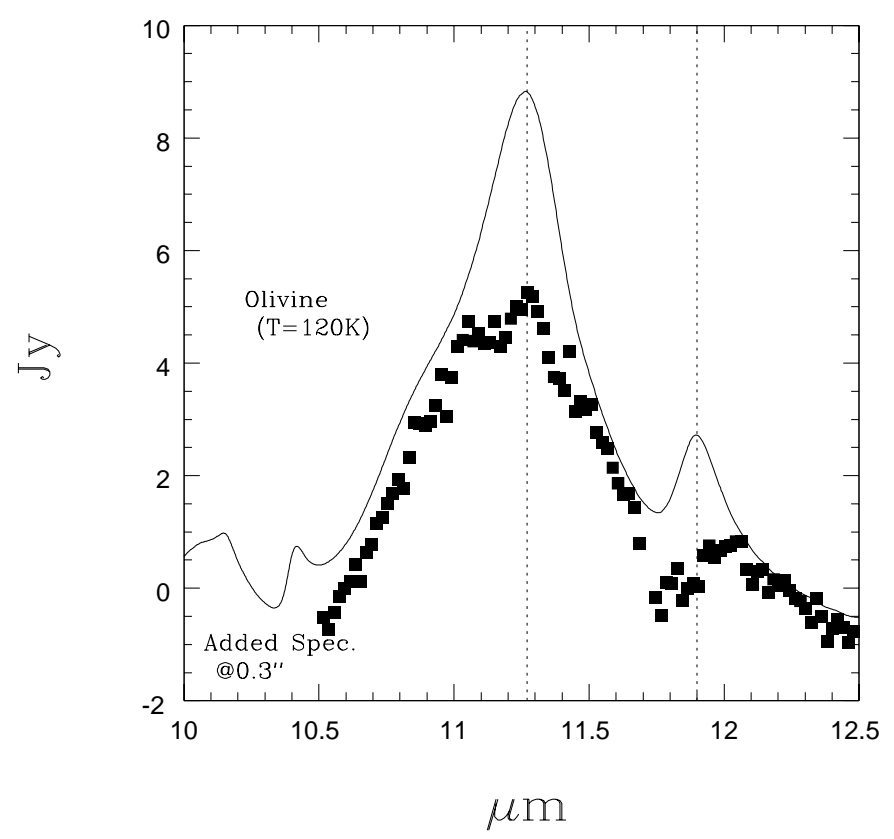

Fig. 8. Summation of the spectra at $0.3^{\prime \prime}$ north, south, east, and west (filled rectangles). The expected intensity of a crystalline olivine $11.3 \mu \mathrm{m}$ feature is shown by a thin line. The temperature of the olivine is assumed to be $120 \mathrm{~K}$ (Waters et al. 1998).

\section{Summary}

We obtained $8.8 \mu \mathrm{m}, 9.8 \mu \mathrm{m}$, and $12.4 \mu \mathrm{m}$ images and lowresolution $N$-band spectra of the Red Rectangle nebula. Both in the imaging and the spectroscopy, we attained diffractionlimited spatial resolution with the 8.2-m SUBARU telescope.

The following is a summary of this study.

(1) The central core is slightly extended at $N$-band wavelengths. The radius of the torus is estimated to be $\sim 0.09^{\prime \prime}$, which corresponds to $\sim 60 \mathrm{AU}$. The core spectrum can be approximated by blackbody emission with a temperature of $358 \mathrm{~K}$. The size and temperature are in good agreement with the predictions of the compact, axisymmetric circumbinary torus model of Men'shchikov et al. (2002).

(2) The UIR emission features at $8.6 \mu \mathrm{m}$ and $11.2 \mu \mathrm{m}$ are prominently observed in the outer region $\left(>0.3^{\prime \prime}\right)$ of the north/south nebula. The peak wavelength of the UIR emissions shifts slightly to shorter wavelengths with increasing distance from the central star. This may be attributed to the isotopic shift due to ${ }^{13} \mathrm{C}$ in the band carriers.

(3) A broad feature peaking at $11.27 \mu \mathrm{m}$ is seen at a distance of $0.3^{\prime \prime}$ (200 AU) in all directions within the nebula. The profile and integrated intensity of the feature are in good agreement with those expected for a crystalline olivine feature of the measured temperature and observed $20 \mu \mathrm{m}$ spectrum. The feature is not seen at the core position, suggesting that crystalline silicates are present only around the dense torus, with the silicate grains in the torus growing too large to emit the feature effectively.

Acknowledgements. The authors would like to thank all of the staff members of the SUBARU telescope for their continued help and support. We also thank S. Wada for helpful suggestions and comments. 


\section{References}

Balser, D. S., McMullin, J. P., \& Wilson, T. L. 2002, ApJ, 572, 326 Bregman, J. D., Rank, D., Temi, P., Hudgins, D., \& Kay, L. 1993, ApJ, 411,794

Cohen, M., Anderson, C. M., Cowley, A., et al. 1975, ApJ, 196, 179

Cohen, M., Walker, R. G., Carter, B., et al. 1999, AJ, 117, 1864

Hony, S., van Kerckhoven, C., Peeters, E., et al. 2001, A\&A, 370, 1030

Hora, J. H., Deutsch, L. K., Hoffmann, W. F., \& Fazio, G. G. 1996, ApJ, 112, 2064

Jura, M., Balm, S. P., \& Kahane, C. 1995, ApJ, 453, 721

Jura, M., Turner, J., \& Balm, S. P. 1997, ApJ, 474, 741

Kataza, H., Okamoto, Y., Onaka, T., et al. 2000, Proc. SPIE, 4008, 132

Kerr, T. H., Hurst, M. E., Miles, J. R., \& Sarre, P. J. 1999, MNRAS, 303, 446

Koike, C., Shibai, H., \& Tuchiyama, A. 1993, MNRAS, 264, 654

Mekarnia, D., Rouan, D., Tessier, E., Dougados, C., \& Lefevre, J. 1998, A\&A, 336, 648

Men'shchikov, A. B., Balega, Y. Y., Osterbart, R., \& Weigelt, G. 1998, New Astron., 3, 601

Men'shchikov, A. B., Schertl, D., Tuthill, P. G., Weigelt, G., \& Yungelson, L. R. 2002, A\&A, 397, 867
Murakawa, K., Tamura, M., Suto, H., et al. 2003, Proc. SPIE, 4843, 196

Okamoto, Y. K., Kataza, H., Yamashita, T., et al. 2003, Proc SPIE, 4841,169

Osterbart, R., Langer, N., \& Weigelt, G. 1997, A\&A, 325, 609

Peerers, E., Hony, S., van Kerckhoven, C., et al. 2002, A\&A, 390, 1089

Reese, M. D., \& Sitko, M. L. 1996, ApJ, 467, L105

Roddier, F., Roddier, C., Graves, J. E., \& Birthcott, M. J. 1995, ApJ, 443, 249

Rowan-Robinson, M., \& Harris, S. 1983, MNRAS, 202, 767

Sakata, A., Wada, S., Tanabe, T., \& Onaka, T. 1984, ApJ, 287, L51

Sloan, C., Grasdalen, G. L., \& LeVan, P. D. 1993, ApJ, 409, 412

Song, I., McCombie, J., Kerr, T., Couch, P., \& Sarre, P. 2003, in preparation

Tuthill, P. G., Men'shchikov, A. B., Schertl, D., et al. 2002, A\&A, 389, 889

van Diedenhoven, B., Peeters, E., van Kerckhoven, C., et al. 2003, in preparation

Wada, S., Onaka, T., Yamamura, I., Murata, Y., \& Tokunaga, T. A. 2003, A\&A, 407, 551

Waters, L. B. F. M., Cami, J., de Jong, T., et al. 1998, Nature 391, 868

Witteborn, F. C., Sandford, S. A., Bregman, J. D., et al. 1989, ApJ, 341,270 